\title{
Cerebellar Tumor Like Lesion in a Patient With Neurosarcoidosis
}

\author{
Bashair Ibrahima, b, Dareen Alshaer $^{\mathrm{a}}$, Omar Ayoub ${ }^{\mathrm{a}}$
}

\begin{abstract}
Sarcoidosis is a systemic granulomatous inflammatory disease that affects multiple organs, most commonly the lungs and lymph nodes. Neurosarcoidosis usually affects the nervous system with no clear systemic involvement. We present a 34-year-old gentleman with left-sided weakness, numbness, and imbalance, with cerebellar tumor like lesion on magnetic resonance imaging (MRI) of the brain. Based on mediastinal lymph node biopsy and after exclusion of other possible differential diagnoses, the patient has confirmed evidence of neurosarcoidosis. He was treated with pulse steroid therapy and subsequent immunosuppression with azathioprine and had excellent response.
\end{abstract}

Keywords: Neurosarcoid; Cerebellar tumor; Sarcoidosis

\section{Introduction}

Neurosarcoidosis is a subtype of sarcoidosis that affects the central and peripheral nervous systems in $5-16 \%$ of the cases. It affects the entire neural axis and can manifest differently according to the structure involved such as cranial nerves, spinal cord, cortical areas, and cerebellum and does not necessarily affect lungs, skin and the eyes that are usually involved in systemic disease [1]. The diagnosis of neurosarcoidosis is difficult, as it necessitates the presence of granuloma in tissue biopsy. Its presentation can be initially mixed up with infection or malignancies of the brain and it is important to differentiate between them due to the impact of the diagnosis on the patient management [2]. Neurosarcoid could present variably as episodic or relapsing forms and is sometimes a mimicker of multiple sclerosis presentation. There is chronic type with a lesion that grows slowly over time, as is the case we are presenting here.

Manuscript submitted July 5, 2017, accepted July 24, 2017

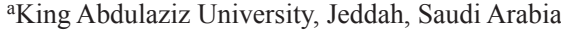

${ }^{b}$ Corresponding Author: Bashair Ibrahim, King Abdulaziz University, Jeddah, Saudi Arabia. Email: bashair.med@gmail.com

doi: https://doi.org/10.14740/jmc2873w

\section{Case Report}

A 34-year-old right-handed gentleman presented to the emergency department (ED) with acute headache and left-sided weakness. He has background history of bronchial asthma, diabetes mellitus (DM), autoimmune thrombocytopenia, and epilepsy.

He presented with acute left occipital headache that is throbbing in nature, progressive over hours, relieved by analgesia but never resolved. It was associated with severe nausea, but no vomiting. He reported left-sided body weakness that is progressive over 5 months with numbness. He had gait instability with ataxic sway to the left side.

He had prior neurological relapses in the form of multiple cranial palsies of the trigeminal, facial nerves and seizures in the years prior to the diagnosis and was labeled as autoimmune disorder without clear cause. In each attack, there was always an associated thrombocytopenia that was avidly responsive to pulses of steroids. Because of his thrombocytopenia, he was maintained on long-term steroid therapy.

Examination showed normal cognition and speech, but right superior quadrantanopia with pallor and atrophy at the nasal part of the right optic disc. Otherwise he had normal cranial nerve examination. He had left-sided hemiparesis in pyramidal distribution and spastic catch at the wrist and elbow, and mildly reduced sensation to pinprick and temperature of the left hemi body with left hemiataxia. Systemic examination showed petechial rash on the left thigh and enlarged lymph nodes in the neck, with normal examination of other body systems.

His basic workup of complete blood count, urea, electrolytes and liver function tests was normal except for the low platelets.

His immunological profile was negative for all variables included: antineutrophil antibody, anti-cytoplasmic antibody, lupus anticoagulants and erythrocyte sedimentation rate. Serology for human immunodeficiency 1 and 2, and hepatitis B and $\mathrm{C}$ was negative.

CSF study showed (Table 1) WBCs of two cells, protein of $0.54 \mathrm{~g} / \mathrm{L}$, glucose of $6.6 \mathrm{mmol} / \mathrm{L}$, with negative gram stain, culture, tuberculosis, and cytology for malignancy.

Carcinogen embryogenic antigen (CEA), alpha fetoprotein, cancer antigen 19-9, and stool for occult blood were negative. 
Table 1. CSF Study of the Patient During Hospital Stay

\begin{tabular}{ll}
\hline WBC & 2 cells \\
Protein & $0.54 \mathrm{~g} / \mathrm{L}$ \\
Glucose & $6.6 \mathrm{mmol} / \mathrm{L}$ \\
PCR mycobacterium & Negative \\
Gram stain & Negative \\
Bacterial culture & Negative \\
Cytology & Lymphocytes \\
\hline
\end{tabular}

MRI of the brain showed abnormal hyperintense signal of the cerebellum that has mass effect and compressing on the fourth ventricle in addition to variable white matter hyperintensities (Figs. 1 and 2).

Computed tomography (CT) scan of the chest showed reticular shadows bilaterally and hilar lymphadenopathy that is compatible with sarcoidosis. There was no evidence of malignancy in CT of chest, abdomen, and pelvis.

Lymph node biopsy showed evidence of granulomatous findings compatible with sarcoid. There were no malignant cells, and Zeihl-Neeisen stain for acid-fast bacilli as well as cultures was negative.

The patient received intravenous methylprednisolone of 1 $\mathrm{g}$ daily for 5 days, followed by intravenous immunoglobulins of $0.4 \mathrm{~g} / \mathrm{kg}$ daily for 5 days.

His clinical status improved significantly with radiological resolution of his cerebellar lesion upon follow-up. He was then maintained on appropriate dose of azathioprine as maintenance therapy in addition to tapering doses of steroids. He was maintained on remission in his 6 and 12 months follow-ups.

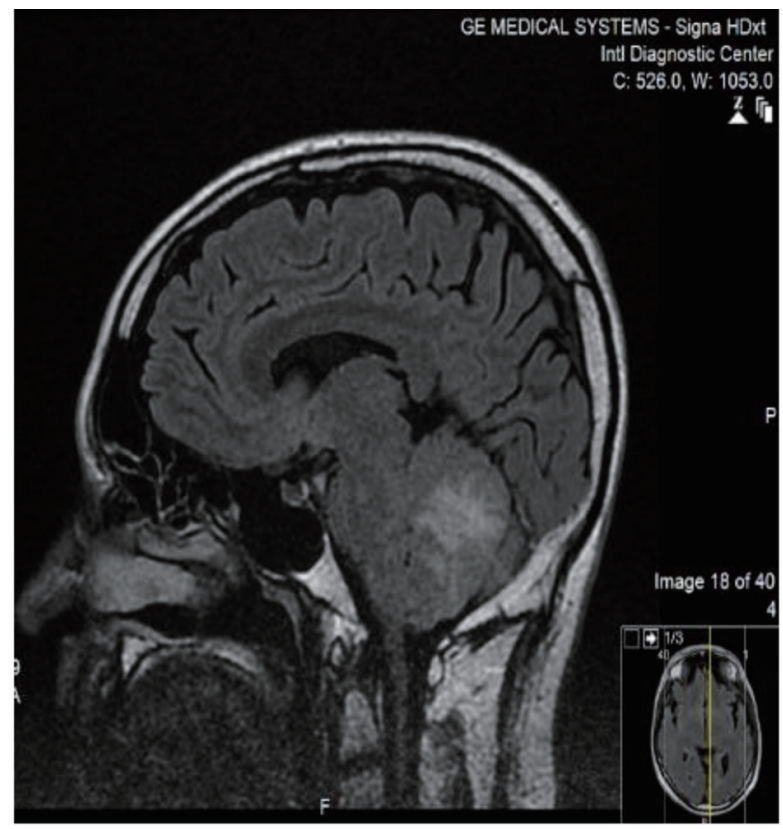

Figure 1. MRI brain/FLAIR sequence of the patient sagittal cut, showing high signal intense lesion in the middle cerebellum, with surrounding edema.

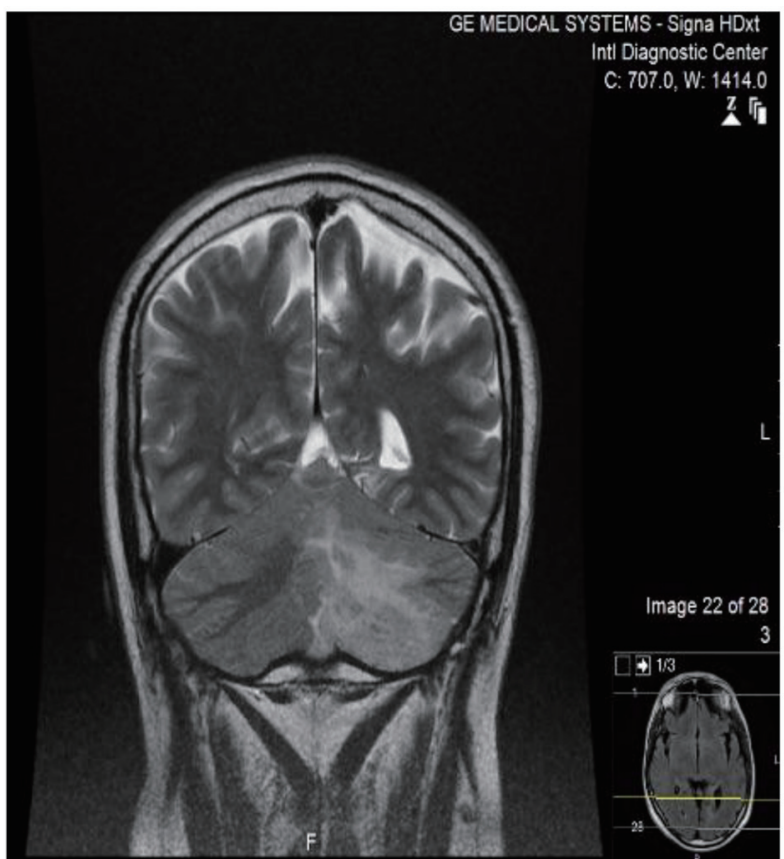

Figure 2. MRI brain/T2 sequence of the patient coronal cut, showing high signal intense lesion in the middle and inferior cerebellum, with surrounding edema, and mild mass effect on the midline.

\section{Discussion}

Neurosarcidosis is a rare disease, which presents in approximately $5-16 \%$ of sarcoidosis patients [1]. Neurosarcoidosis affects the central nervous system (CNS) and peripheral nervous system (PNS) or both, and clinical symptoms and signs manifest according to the structure involved such as, cranial nerves, spinal cord, cortical areas, and cerebellum. It can present with headache, seizure disorder, myelopathy, peripheral neuropathy, neuropsychiatric, and neuroendocrine dysfunction [3]. There is no specific test to diagnose other than confirmation by tissue biopsy [4].

In our case, it mimicked brain tumor and could have been mislabeled as such. After treatment the patient improved dramatically and we did not need to start the patient on infliximab as the next management line [5].

Upon follow-up, he maintained to be in remission on moderate doses of steroids and azathioprine.

Here we present the first case of neurosarcoidosis from Saudi Arabia. It is unknown how common is the disease prevalent in our population and more studies are needed to explore that.

Our case is peculiar in many aspects. First, the diagnosis was delayed for sometime as he was labeled as autoimmune thrombocytopenia with multiple cranial neuropathies before the diagnosis was made. Clinicians have to put sarcoidosis in their differential of inflammatory like presentation like our patient.

Second, he had systemic manifestations in addition to the CNS features evident by his lymphadenopathy and radiological involvement of his chest CT scan. 
Third, we think that his seizure disorder was directly related to CNS involvement as he was in remission with immunosuppression over the months of follow-up.

We advocate investigating for sarcoidosis in any inflammatory/tumor like lesion of the brain and obtaining more tissue biopsy in order to prove the diagnosis. Because of the rarity of the disease, we depend on such case reports to document the existence of the disease in our patients.

\section{Reference}

1. Lower EE, Broderick JP, Brott TG, Baughman RP. Diagnosis and management of neurological sarcoidosis. Arch
Intern Med. 1997;157(16):1864-1868.

2. Malik WT, Syed NM, Khan HH, Malik MB, Khan GH, Kazi SA. Noncaseating granulomatous disease involving the meninges: a case report. Kasr Al Ainy Medical Journal. 2016;22(3):153.

3. Nozaki K, Judson MA. Neurosarcoidosis. Curr Treat Options Neurol. 2013;15(4):492-504.

4. Bagnato F, Stern BJ. Neurosarcoidosis: diagnosis, therapy and biomarkers. Expert Rev Neurother. 2015;15(5):533548.

5. Tana C, Wegener S, Borys E, Pambuccian S, Tchernev G, Tana M, Giamberardino MA, et al. Challenges in the diagnosis and treatment of neurosarcoidosis. Ann Med. 2015;47(7):576-591. 